\title{
A note on groups of intermediate growth
}

\author{
B. Bajorska and O. Macedońska \\ Institute of Mathematics, Silesian University of Technology, \\ Gliwice 44-100, Poland \\ e-mail: b.bajorska@polsl.pl,o.macedonska@polsl.pl
}

\begin{abstract}
It was proved by R. I. Grigorchuk in 1983 that there exist groups which are neither of polynomial nor of exponential growth. Their growth is called intermediate. We show that every group of intermediate growth has either a residually finite quotient of intermediate growth or a simple section of intermediate growth.
\end{abstract}

Keywords: Groups of intermediate growth; Residually finite groups. 2000 AMS subject classification: 20E07. 


\section{INTRODUCTION}

The notion of growth was introduced for algebras first. For groups, following Milnor (1968a), the definition can be given as:

Let $G$ be a group generated by a finite set $S$ and for every natural $n$ let $g_{(G, S)}(n)$ be the number of distinct elements in $G$ that can be expressed as products of at most $n$ elements from $S \cup S^{-1}$.

- The group $G$ is of polynomial growth if there exist real numbers $d, C$ such that $\forall n \in \mathbb{N} \quad g_{(G, S)}(n) \leq C n^{d}$.

- The group $G$ is of exponential growth if there exists a real number $C>1$ such that $\forall n \in \mathbb{N} \quad g_{(G, S)}(n) \geq C^{n}$.

The notion of growth does not depend on the choice of a finite generating set, as shown by Milnor (1968a, Lemma 1). Since it is defined for finitely generated groups, in further text we will consider only such groups and sometimes omit the words "finitely generated".

Let us also establish, that if $P_{1}$ and $P_{2}$ are group-theoretical properties, then a group $G$ will be called $P_{1}$-by- $P_{2}$ if and only if $G$ contains a normal subgroup $N$ satisfying the property $P_{1}$ such that $G / N$ satisfies $P_{2}$.

Combining the results of Gromov (1981) and Wolf (1968, Theorem 3.2) we conclude that a finitely generated group $G$ is of polynomial growth if and only if it is nilpotent-by-finite.

Milnor (1968b) and Wolf (1968) proved that every finitely generated soluble-by-finite group has either polynomial or exponential growth. In the context of this result Milnor, in a joint work with Power et al. (1968, Problem 5603), formulated the famous question whether this dichotomy holds for all groups. Tits (1972) showed that it is true for linear groups. However Grigorchuk (1983) constructed examples answering this question negatively. Since then every group whose growth is neither polynomial nor exponential is called a group of intermediate growth. It was acknowledged by R. I. Grigorchuk in 1984 that S. V. Aleshin had already found such groups in 1971 to settle the Burnside problem (via finite automata).

Groups of intermediate growth were found by many authors and all of them were residually finite. This motivated Grigorchuk (1989) to raise the famous conjecture saying that every group of intermediate growth is residually finite. Examples showing that it does not hold were constructed by Erschler (2004). However, Erschler's groups have residually finite quotients of intermediate growth and one may ask whether this property always holds. 
In this note we show that every group of intermediate growth either has a residually finite quotient of intermediate growth or has a simple section of intermediate growth.

\section{THE THEOREM}

We start with some useful results.

Let $G$ be a group and let $G \geq H \unrhd N$. Then $H / N$ is called a section of $G$. If $H=G$, the section $G / N$ is called a quotient of $G$. The definition of growth immediately implies the following

Proposition 1 If $G$ is a group of intermediate growth then every finitely generated section of $G$ is either of polynomial or of intermediate growth.

Proposition 2 Every finite-by-nilpotent group is nilpotent-by-finite.

Proof. Let $G$ be a group and let $N$ be its finite normal subgroup such that $G / N$ is nilpotent of class $c$, that is $(G / N)_{(c+1)}=1$, in notation of Neumann $(1967,14.64)$. Thus $G_{(c+1)} \leq N$. The centralizer $C$ of $N$ in $G$ is obviously a normal subgroup. Moreover, since $N$ is finite, all conjugacy classes of elements in $N$ are finite, whence the centralizers of all elements in $N$ have finite indices. Therefore the centralizer $C$, as their intersection, has a finite index as well. Furthermore, $C_{(c+2)}=\left[C_{(c+1)}, C\right] \leq[N, C]=1$, so $C$ is nilpotent. Thus $C$ is a nilpotent normal subgroup of finite index in $G$, which means that $G$ is nilpotent-by-finite as required.

Since multiplication of varieties is associative (see Neumann $(1967,21.51)$ ) and every nilpotent group is solvable, from Proposition 2 we deduce the following

Corollary 1 (1) Each (solvable-by-finite)-by-(nilpotent-by-finite) group is solvable-by-finite.

(2) Each finite-by-(nilpotent-by-finite) group is nilpotent-by-finite.

Proposition 3 If $G$ is a group of intermediate growth having a nontrivial quotient $G / R$ of polynomial growth, then $R$ is a finitely generated group of intermediate growth. 
Proof. If $G$ is a group of intermediate growth (whence finitely generated) and $G / R$ is of polynomial growth, then obviously $R \neq 1$. We prove first that $R$ is finitely generated.

Note that $G / R$, being of polynomial growth, is nilpotent-by-finite by Gromov (1981), and hence contains a nilpotent subgroup $H / R$ of finite index. Next, since $|G: H|=|(G / R):(H / R)|<\infty$ and $G$ is finitely generated, both $H$ and $H / R$ are finitely generated. As a finitely generated nilpotent group, $H / R$ is polycyclic (see Neumann $(1967,31.12)$ ), thus there exists a finite subnormal series $H=H_{0} \triangleright H_{1} \triangleright \cdots \triangleright H_{m}=R$ with all factors $H_{i-1} / H_{i}$ cyclic.

Observe now that $G$ cannot contain a free nonabelian subsemigroup (because otherwise it would be of exponential growth). Moreover, $H_{0}$ is finitely generated and $H_{0} / H_{1}$ is cyclic. Hence combining Lemma 1 and Lemma 2 of Longobardi et al. (1995), we obtain that $H_{1}$ is finitely generated. Proceeding in this way for each $i=2, \ldots, m$ we conclude that each $H_{i}$ and whence finally $R$ is finitely generated as required.

Now, by Proposition $1, R$ is either of intermediate or of polynomial growth. If $R$ is of polynomial growth then $G$ is (nilpotent-by-finite)-by(nilpotent-by-finite), so by Corollary 1 (1), $G$ is solvable-by-finite. Then by Milnor (1968b) and Wolf (1968), $G$ is either of polynomial or of exponential growth, a contradiction. Therefore $R$ is a finitely generated group of intermediate growth which finishes the proof.

Theorem Let $G$ be a group of intermediate growth and let $R$ denote the intersection of all normal subgroups of finite index in $G$. Then at least one of the following conditions holds

(i) $G / R$ is a residually finite group of intermediate growth,

(ii) $R$ has a simple quotient of intermediate growth.

Proof. Let $G$ and $R$ satisfy the assumptions. Note that $G$ is finitely generated. If $G / R$ is a group of intermediate growth then the condition $(i)$ holds.

In view of Proposition 1, we can assume now that $G / R$ is a group of polynomial growth (whence nilpotent-by-finite). We prove first that $R$ does not contain a proper normal subgroup of finite index. If $R=G$, it follows from the definition of $R$. If $R \neq G$, then by Proposition $3, R$ is a finitely generated group of intermediate growth. Suppose that $T$ is a normal subgroup of finite index in $R$. Then $T$ contains a subgroup $K$ characteristic in $R$ and of finite index in $R$ (Lyndon and Schupp (2000, Theorem IV.4.7)). Since $K$ is characteristic in $R$ and $R$ is normal in $G$, also $K$ is normal in $G$. Now, since 
$R / K$ is finite and $G / R$ is nilpotent-by-finite, the isomorphism $(G / K) /(R / K)$ $\cong G / R$ implies that $G / K$ is finite-by-(nilpotent-by-finite), whence nilpotentby-finite (by Corollary $1(2)$ ). Since nilpotent-by-finite groups are residually finite (Hall, 1959), then $G / K$ is residually finite. Consequently $R \leq K$, whence $K=R$ and finally $T=R$. Therefore $R$ does not contain a proper normal subgroup of finite index.

Let now $N$ be a maximal proper normal subgroup of $R$, which exists in every finitely generated group by Zorn's Lemma. Then $R / N$ is a finitely generated infinite simple group, therefore it cannot be nilpotent-by-finite. Thus it cannot be of polynomial growth and consequently (by Proposition 1) $R$ has a simple quotient of intermediate growth which means that the condition (ii) holds.

The result can be formulated in not so detailed form as

Corollary 2 Every group of intermediate growth has either a residually finite quotient of intermediate growth or a simple section of intermediate growth.

Remark Groups constructed by Erschler (2004) satisfy the condition $(i)$ in the Theorem. However, it is an open problem whether there exist groups satisfying the condition $(i i)$.

\section{REFERENCES}

Erschler, A. (2004). Not residually finite groups of intermediate growth, commensurability and non-geometricity. J. Algebra 272:154-172.

Grigorchuk, R. I. (1983). On the Milnor problem of group growth (Russian). Dokl. Akad. Nauk SSSR 271(1):30-33.

English translation: (1983). Soviet Math. Dokl. 28(1):23-26.

Grigorchuk, R. I., Kurchanov, P. F. (1989). Some questions of group theory related to geometry (Russian). Itogi Nauki i Tekhniki, VINITI Akad. Nauk SSSR. Moscow.

English translation: (1993) Part II of Algebra VII. Combinatorial group theory. Applications to geometry. Springer-Verlag, New York.

Gromov, M. (1981) Groups of polynomial growth and expanding maps. Inst. Hautes Études Sci. Publ. Math. 53:53-78. 
Hall, P. (1959) On the finiteness of certain soluble groups. Proc. London Math. Soc. 9(3):595-622.

Longobardi, P., Maj, M., Rhemtulla, A. H. (1995) Groups with no free subsemigroups. Trans. Am. Math. Soc. 347:1419-1427.

Lyndon, R. C., Schupp, P. E. (2001) Combinatorial group theory. 2nd ed. Berlin Heidelberg. Springer-Verlag.

Neumann, H. (1967). Varieties of groups. Berlin-Heidelberg-New York. Springer-Verlag.

Milnor, J. (1968a). A note on curvature and fundamental group. J. Diff. Geom. 2:1-7.

Milnor, J. (1968b). Growth of finitely generated solvable groups. J. Diff. Geom. 2:447-449.

Power, E. A., Milnor, J., Carlitz, L., Struble, R. A., Shafer, R. E., Wilansky, A., Maas, R. E., Felsinger, N., Simoes, J. M. S. (1968). Problems and solutions: Advanced problems. Amer. Math. Monthly 75(6):685-687.

Tits, J. (1972). Free subgroups in linear groups. J. Algebra 20:250-270.

Wolf, J. A. (1968). Growth of finitely generated solvable groups and curvature of riemannian manifolds. J. Diff. Geom. 2:421-446. 